\title{
Great East Japan earthquake: anesthetists in Minamisoma
}

\author{
Masahiko Akatsu ${ }^{1} \cdot$ Chiaki Nemoto $^{2} \cdot$ Yukihiro Ikegami $^{3}$
}

Received: 27 October 2015 / Accepted: 12 November 2015 / Published online: 8 December 2015

(C) Japanese Society of Anesthesiologists 2015

To the Editor:

Minamisoma Municipal General Hospital is located along the coast of Fukushima Prefecture, $23 \mathrm{~km}$ from the Fukushima Daiichi Nuclear Power Plant. We monitored air radiation levels, the number of cases managed by anesthesiologists, and reconstruction efforts for the period 2010-2014 (1 year pre-disaster, 4 years post-disaster). Immediately after the catastrophic breakdown of the Fukushima Daiichi Nuclear Power Plant, air radiation levels were $20.00 \mu \mathrm{Sv} / \mathrm{h}$ immediately outside the hospital and $2.50 \mu \mathrm{Sv} / \mathrm{h}$ indoors. The hospital had no anesthesiologists at the time of the earthquake, although the number of cases managed by anesthesiologists at the present time is now back to previous levels (Electronic Supplementary Material Table 1). There remains a lack of hospital beds and staff, but efforts are being made to reduce the lengths of stay of patients admitted to the hospital. The hospital and its staff face numerous problems: direct earthquake damage; effects of long-term radiation exposure; economic damage; misinformation; ethical issues; social changes; financial difficulties;

Electronic supplementary material The online version of this article (doi:10.1007/s00540-015-2106-9) contains supplementary material, which is available to authorized users.

Masahiko Akatsu

masahikoakatau@hotmail.co.jp

1 Department of Anesthesiology, Minamisoma Municipal General Hospital, 54-6 Takamicho 2-chome, Haramachi-ku, Minamisoma, Fukushima 975-0033, Japan

2 Department of Emergency and Critical Care Medicine, School of Medicine, Fukushima Medical University, Fukushima, Japan

3 Department of Anesthesiology, School of Medicine, Fukushima Medical University, Fukushima, Japan changes in public health policies; mental health issues; general illness; legal problems [1]. These problems particularly affect the elderly and children. Air radiation levels have decreased through decontamination efforts, but the local population is declining. Social changes include a decrease in the number of children and able workers [2]. We are concerned about the effects of radiation on the children of hospital employees which has the potential to affect staffing levels and lead to fewer hospital beds. If the present trend continues, we will be unable to increase the number of hospital anesthesiologists and hence the number of surgeries performed. The average age of our anesthesiologists is 58.5 years at this time and is progressively increasing. We are very concerned about this situation, which amounts to a medical crisis.

Compliance with ethical standards

Conflict of interest None.

\section{References}

1. Ikegami Y, Konno S, Isosu T, Obara S, Hakozaki T, Akatsu M, Murakawa M. Current state of emergency medicine in Fukushima 4 years after the Great East Japan earthquake. Emerg Med J. 2015;32:665-7.

2. General Affairs Department Secretary Division Minamisoma. Available at: http://www.city.minamisoma.lg.jp/. Accessed 1 Oct 2015. 